\title{
DOM AND DEM SYNCHRONIZATION EDITIONG AND UPDATING METHOD BASED ON GEOMORPHOLOGICAL CHANGE DETECTION
}

\author{
Cai Zhigang ${ }^{1}$, Xiang Zhiyong ${ }^{1}$, Zhang Li $^{2}$, Liu Weiwei ${ }^{1}$ \\ ${ }^{1}$ Zhejiang First Surveying and Mapping Institute, Hangzhou 310012, China \\ ${ }^{2}$ Chinese Academy of Surveying\&Mapping, Beijing 100830, China
}

KEY WORDS: DOM, DEM, Geomorphological Change Detection, Synchronization Editing and Updating Method

\begin{abstract}
:
Aiming at the deficiency of the existing DOM and DEM production modes and the networking and automation requirements of the information mapping production system, a method of network-based simultaneous editing and updating of DOM and DEM based on geomorphological change detection was proposed, a set of rapid update technical processes for changing regions were formed, and the DOM and DEM network rapid editing and updating platform was developed. The technology process is based on network parallel environment to achieve network task distribution and multi-person synchronous editing production, and the production efficiency of DOM and DEM is greatly improved.
\end{abstract}

\section{ITRODUCTION}

Since 2014, the quasi dynamic updating model was officially launched to update 1:10000 fundamental geographic information digital products by Zhejiang Administration of Surveying Mapping and Geo-information. According to this model, the key elements would be updated within three months, the important geographic elements would be updated within six months, and the others would be updated within one year (abbreviated as "361" update). The start of this model puts higher demands on the production efficiency of 3D (DLG, DOM, DEM) products (Shaoqin Chen, 2013). However, the existing technology and production mode of DOM and DEM is only suitable to solve the "from scratch" production needs, and the update production will cause a lot of human and financial waste. A new more flexible, faster and more effective way is required to quickly discover changes (Jianya Gong, Qing Zhu , 2007) and quickly update DOM and DEM (Qing Zhu, Wenzhong Shi, 2013).

At present, there are a lot of DOM and DEM production softwares, such as JX4(Aihong Kong, 2012), VirtuoZo, INPHO, DPGrid (Yang Fu, Jiyong Liu, Hongyan LI, 2012), PixelFactory, etc. Although they have large differences in software functions and work patterns, they almost have a similar technical route: manual editing DEM, ortho rectification, image mosaic, image editing (based on image editing software, such as Photoshop and so on), image subset and cutting. Most of them are carried out in the mode of single machine operation with partitioned blocks, and the different working procedures are implemented in different work modules. As a result, many problems arise, such as tedious process, long period of work, low degree of networking and automation and so on.

Therefore, aiming at the existing problems of DOM and DEM production mode, based on the previous research, this paper proposes a set of change detection technology based on multitemporal (Xiaodong Zhang,2005), multi-scale (S. Magnussen, $\mathrm{P}$ Boudewyn, M. Wulder, 2004), and multiple data sources (M. Baatz, A. Schape, 1999).Then a networked synchronous editing and updating method of DOM and DEM based on geomorphic change detection was proposed, and a DOM and DEM quickly production network platform was developed. The technology process is based on network parallel environment to achieve network task distribution and multi-person synchronous editing production, and the production efficiency of DOM and DEM are greatly improved. The following works have been completed (Guobin Zhu, 2003).

\section{EXTRACTION OF GEOMORPHIC CHANGE BASED ON OBJECT-ORIENTED METHOD}

The surface changes caused by human activities are often closely related to roads and water systems. Taking road and river system change as entry point, object oriented method ( Bao Cao, et al, 2006). is used to extract thematic information, mainly including multi-scale segmentation, building knowledge rule base, automatic classification and human-machine interaction processing.

Then the data of the two periods are analyzed by space superposition, and the change information can be obtained. To use the two characteristics of area and ratio of perimeter to area to remove the small or slender polygon, the accuracy of the change results are greatly improved.

\subsection{Multi-scale Segmentation}

In multi-scale segmentation, image segmentation using old road edges as thematic information can better maintain the original road features and enhance the segmentation effect. Because the spectral features of the water body in the image are obvious and the shape features are diverse, the spectral features should be highlighted as much as possible. Split parameters are as follows:

\begin{tabular}{l|l|l|l|l}
\hline & \multirow{2}{*}{$\begin{array}{l}\text { Segmentat } \\
\text { ion Scale }\end{array}$} & $\begin{array}{l}\text { Color } \\
\text { Factor }\end{array}$ & \multicolumn{2}{|l}{ Shape Factor } \\
\cline { 4 - 5 } & & Compactness & $\begin{array}{l}\text { Smooth } \\
\text { ness }\end{array}$ \\
\hline Road & 25 & 0.8 & 0.3 & 0.7 \\
\hline River & 40 & 0.9 & 0.6 & 0.4 \\
\hline
\end{tabular}

Table 1. Split Parameters 


\subsection{Establishing a Knowledge Rule Base}

Based on the segmented objects, the classification rules are constructed through feature analysis and extraction. The characteristics of road extraction in the experiment are as follows:

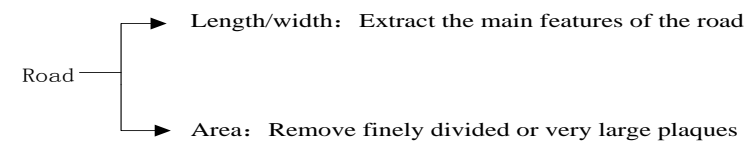

Figure 1. Road Extraction Features

The spectral characteristics of water are obvious, because water absorbs more light in the near-infrared region, so the spectral values in the near-infrared region are generally low, and the reflectance in the blue and green wavebands is relatively high. Water can be distinguished based on these characteristics. The formula of Water Index $(W I)$ is as follows:

$$
W I=\frac{\text { meangreen }- \text { meannir }}{\text { meangreen }+ \text { meannir }}
$$

However, there are also large differences in the water bodies in the study area. For example, the shapes of lakes and rivers are very different, and the same lakes have different spectral differences due to different reflections in different regions. This intra-class divergence has made it difficult to extract features using a classification rule. Therefore, the water body is further subdivided into a number of sub-categories, which establish rule extractions and finally classify them into a large category. According to the characteristics of the water in the study area, the water body is extracted according to the following rules:

\begin{tabular}{c|l|l}
\hline Class & Rules & Notes \\
\hline Water1 & WI, NDVI & $\begin{array}{l}\text { In general, the NDVI of a } \\
\text { water body is less than 0, } \\
\text { which may distinguish water } \\
\text { bodies from part of mountain } \\
\text { shadows. }\end{array}$ \\
\hline Water2 & $\begin{array}{l}\text { WI, standard } \\
\text { deviation pan }\end{array}$ & $\begin{array}{l}\text { Extraction of water bodies } \\
\text { subject to artificial } \\
\text { disturbance and relatively } \\
\text { severe cloud cover along the } \\
\text { coast }\end{array}$ \\
\hline Water3 & $\begin{array}{l}\text { Brightness, NDVI, } \\
\text { WI, Surrounded by } \\
\text { Water1 }\end{array}$ & $\begin{array}{l}\text { Brightness, NDVI, } \\
\text { WI, Ratio of length } \\
\text { to width, boundary } \\
\text { have already extracted the } \\
\text { vast majority of water bodies, } \\
\text { and "Water 3" and "Water 4" } \\
\text { are for finer extraction of less } \\
\text { partial leakage of the water }\end{array}$ \\
\hline
\end{tabular}

Table 2. Rules of Water Extraction

\subsection{Automated Classification and Human-computer Interaction Processing}

After the classification rules are established, the classification rule sets are automatically classified to obtain the results of automated classification. eCogniton provides such functions as object block merging, object block segmentation, object category attribute change, etc, which can facilitate visual judgment of misclassified objects, manual classification, adjustment of classification results and improvement of classification accuracy.

\subsection{Change Detection}

The two-period thematic information extraction datum were analyzed by spatial superposition to obtain change information. In addition, due to error transmission of image geometric correction and the deviation of the classification results, a large number of narrow and long fine patches will appear as a result area, the results are processed and the most finely and elongated patches are removed, and the accuracy and translatability of the results are greatly improved.

\section{METHOD OF DOM AND DEM NETWORK SYNCHRONIZATION EDITING AND UPDATING}

The production of DOM depends heavily on DEM. However, in actual production, their production processes were separated. The DEM correction could not be realized on the DOM in real time. It is often necessary to repeatedly edit, modify and correct images on multiple software platforms to achieve the desired effect. At the same time, the existing DEM and DOM updating was still based on the single-machine and single-frame production model, thus leading to a lot of edge-to-edge work and substantial management and storage costs. This article proposed the production process and platform of synchronous updating technology for DOM and DEM.

\subsection{Management of Network-based Distributed Production Resource}

In the unit of measurement area, the resource data needed to update DEM and DOM are managed in a unified manner to achieve sharing based on network. Tasks are divided through the network, so that production persons can quickly acquire tasks. Different production persons can edit and update DEM and DOM synchronously, and they do not need to deliberately connect the edges. When framing results are needed, the entire final product can be cut separately.

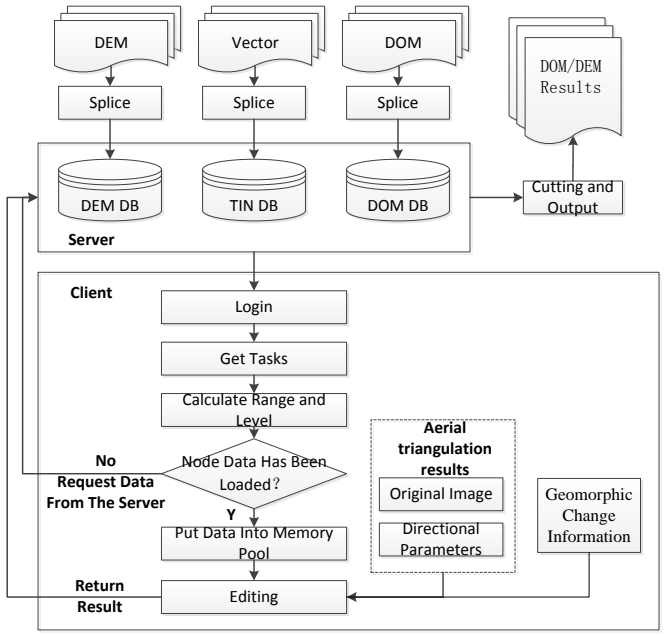

Figure 2. DOM and DEM Update Production Network Distributed Management 


\subsection{Synchronized Editing and Update of DOM and DEM}

Through the synchronized editing and updating of DOM and DEM to achieve visual editing of DEM, DOM can be corrected in real time. On the one hand, repeated switching between different softwares can be avoided, and the efficiency is greatly improved. On the other hand, it can be processed in time to deal with the DOM deflection and distortion caused by some elements (such as viaduct, house, etc.) not presented in DEM. The effect is as shown below.

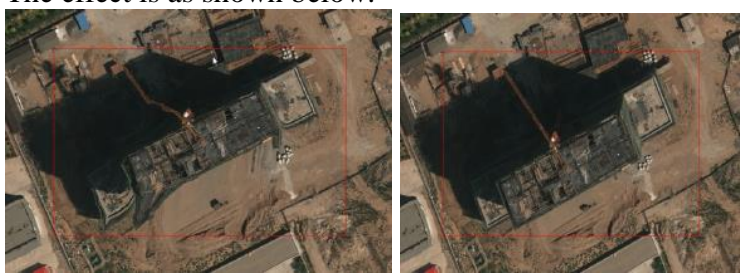

a. Housing distortion

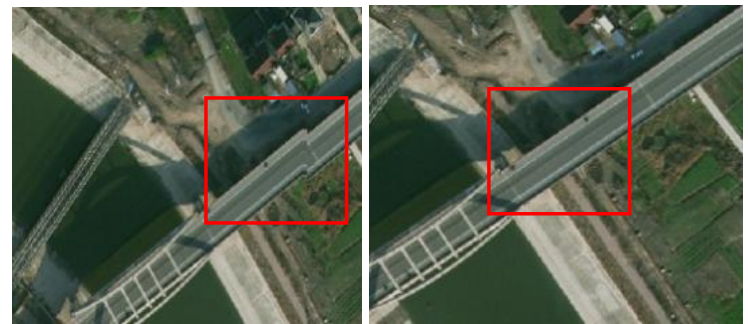

b. Bridge deformation

Figure 3. Effect of DOM and DEM Synchronized Editing Function

\subsection{DOM and DEM Rapid Production Updating Technolo- gy Process}

Based on the idea of updating the elements of change, a set of DOM and DEM network-based synchronous editing and updating technology processes are proposed as follows:

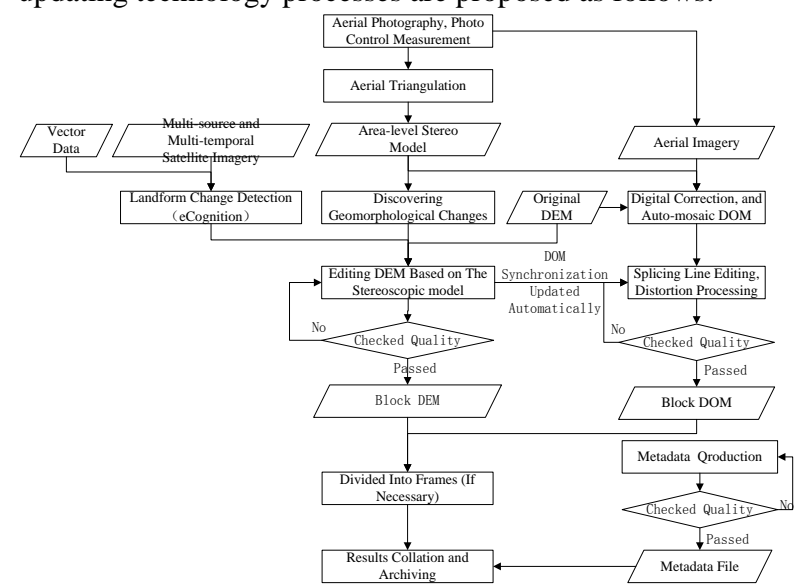

Figure 4. DOM and DEM Rapid Updating Technology Process

\section{EXPERIMENT}

\subsection{Change Detection Test}

4.1.1 Data source: Data I: SPOT5 multi-spectral and panchromatic images (February 2009 and December 2011) with a multi-spectral band resolution of 10 meters and a full-color band resolution of 2.5 meters. This datum will be used as source data for water extraction.

Data II: 1:10000 DLG data (October 2011). Based on this data, road sidelines and structural lines were extracted.

Data III: Color aerial images (September 2012) corrected and made into DOM with $0.5 \mathrm{~m}$ resolution. Use this images as the source data for road extraction.

Data pre-processing includes image correction and registration and image contrast enhancement. The SPOT5 multi-spectral and panchromatic bands were corrected by reference to DOM respectively.

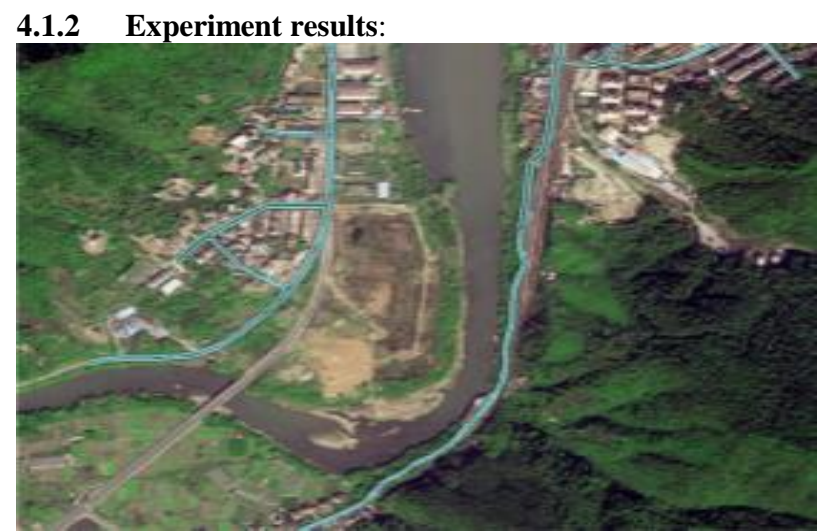

(a). Original data

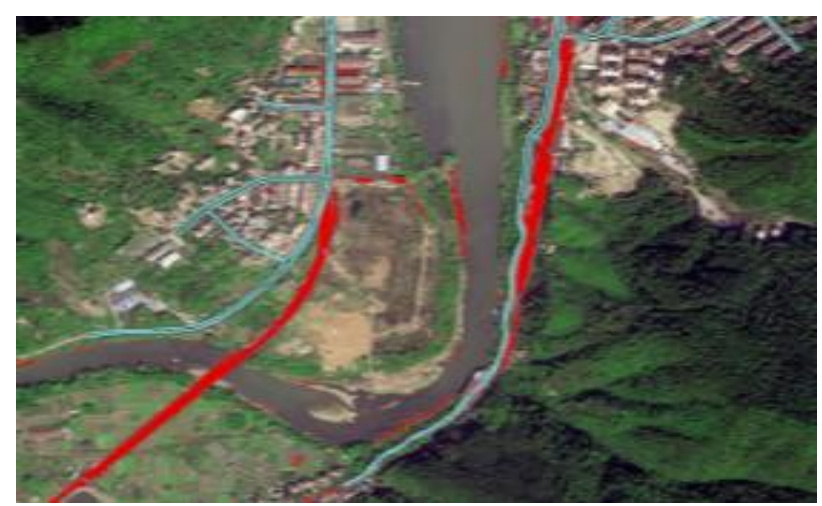

(b). Changed Road Extraction (shown in red)

Figure 5. Changed Road Extraction Results 


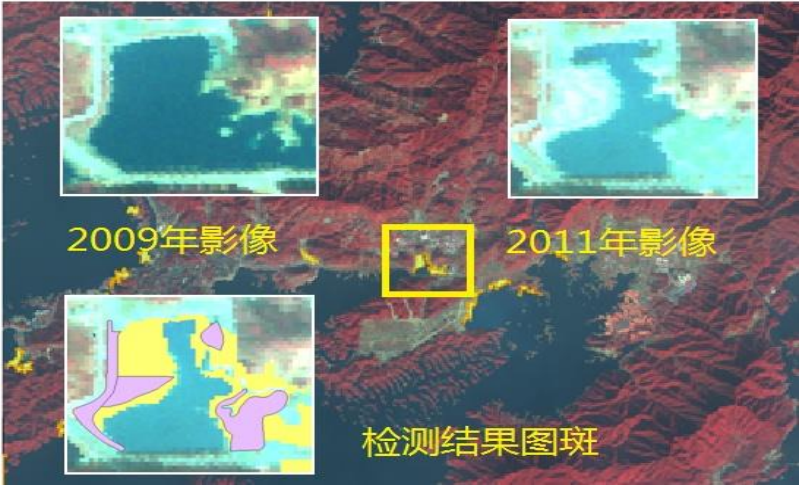

Figure 6. Changed Water Detection Results

\subsection{DOM/DEM Synchronization Editing Experiment}

4.2.1 Test data: Data I: The aerial photography taken in September 2012 used aerial camera for ULTRACAMX with focal length of $100.5 \mathrm{~mm}$ and ground resolution of $0.35 \mathrm{M}$. It is used to update the 1:10000 DEM (grid 5m) and DOM (resolution $0.5 \mathrm{~m}$ ) in the measuring area, involving 77 images of 1:10,000 images(about 2140 sq kilometers). The survey area is dominated by mountains and hilly landscapes, and the terrain is tilted from southwest to northeast. The southwestern region is mainly dominated by medium height mountains with low mountain, hills and valleys, while the northeast is mainly low mountains as well as valley basins. In the experiment, the previous generation DLG and DEM with a scale of 1:10000 were used to assist production and update.

Data II:The aerial photography taken in January 2014 used aerial camera UCXP-WA with focal length of $70 \mathrm{~mm}$ and ground resolution of $0.18 \mathrm{M}$, covering an area of $537 \mathrm{sq} \mathrm{km}$. These images were used for 1:2000 DOM production for digital urban geo-spatial framework. The survey area is about $537 \mathrm{sq}$ kilometers, located in the northeast of the Hangjiahu Plain, which is a typical plain area with an average elevation of 2.8 meters, where the distribution of water network systems is intensive. LIDAR data (with a grid spacing of $2 \mathrm{~m}$ ) and DLG vector geomorphological data were used as source data to assist the production of DOM.

4.2.2 Test results: The results of the two test areas have passed the inspection of Zhejiang Quality Inspection Institute of Surveying and Mapping Achievements, and the accuracy is in full compliance with the requirements of the relevant national regulations of 1:10000, 1:2000 DEM and DOM. The overall efficiency is about $27 \%$ higher than traditional production methods.

Through the production practice in two test areas, it is verified that the modified technology and newly developed platform are suitable for different terrain, such as plain, water network and mountain area, and meet the production requirements for DEM/DOM of different scales. It has obvious advantages compared with traditional models in the aspects of multi-source data source compatibility, massive data support, network organization, synchronous editing, project management and so on.

\section{CONCLUSION}

Based on a thorough study of the existing DOM and DEM updating technology and process flow, the general ideas and technical methods for DOM and DEM synchronization updating were proposed, a rapid updating platform for DOM and DEM synchronization updating was developed, and the production technology process was reconstructed. After trial production in several testing areas, it was proved that the technology has improved the existing DOM and DEM production methods, increased the efficiency and production management level, and made the production of DOM and DEM updating more networked and automated.

At present, the research results have been used in the production practice. From the actual performance, with the improvement of the software proficiency of the workers and the improvement of software platform function, the productivity of DEM and DOM updating still has great potential, and the network updating production based on high-resolution stereo mapping satellite image will be the direction of further research.

\section{REFERENCES}

Aihong Kong, 2012. A brief discussion of production of OrthoPhoto map using the DPGrid software. Geomatics\&Spatial Information Technology, 35(5), pp. 125-127. County,

Bao Cao, Qiming Qin, Haijian Ma, Yunfeng Qiu, 2006. Application of object-oriented approach to SPOT5 image classification: a case study in Haidian district, Beijing city. Geography and Geo-Information Science, 22(2), pp. 46 -54.

Guobin Zhu, 2003. Remote sensing image analysis method for multi-resolution hierarchy. Journal of Wuhan University,28(3), pp.315-320.

Jianya Gong, Qing Zhu , 2007. A brief discussion on the goals and tasks of the construction of information-based surveying and mapping systems. State Key Laboratory of Information Engineering in Surveying, Mapping and Remote Sensing. Wuhan University, China.

M. Baatz, A. Schape, 1999. Object-Oriented and Multi-scale image analysis in semantic networks. Proc of the 2nd International Symposium on Operationalization of Remote Sensing. ITC, Enschede.

Qing Zhu, Wenzhong Shi, 2013. Theories and Methods of Geographical National Conditions Monitoring. Science Press, Beijing, pp.101-121.

Shaoqin Chen, 2013. Reconstruction and Standardization of Map Renewal Production Technology System. Standardization Technical Committee of Zhejiang Administration of Surveying Mapping and Geo-information.

S. Magnussen, P. Boudewyn, M. Wulder, 2004. Contextual Classification of Landsat TM Image to Forest Inventory Covertypes. International Journal of Remote Sensing, 25(12), pp. 2421-2440.

Xiaodong Zhang,2005. Research on the theory and method of change detection based on remote sensing image and GIS. Wuhan University, China.

Yang Fu, Jiyong Liu, Hongyan LI, 2012. Method for improving DOM production with JX4. Urban construction theory research, pp.16. 